minimal and non-significant bias between the two modalities (bias $=0.01 \mathrm{~m} / \mathrm{s} ; \mathrm{p}=0.91$ and $-0.08 \mathrm{~m} / \mathrm{s} ; \mathrm{p}=0.91$ ). This novel automated method demonstrated excellent reproducibility (Coefficient of variability $2.67 \%$ for peak E-wave mitral inflow velocity; Coefficient of variability $1.93 \%$ for peak A-wave mitral inflow velocity).

Conclusion We present a novel automated time-resolved transvalvular peak velocity assessment solution that can be used clinically for mitral inflow assessment and would circumvent the limitations of pulse-wave doppler echocardiography. Future studies are warranted to explore the diagnostic and prognostic advantages of our novel automated technique for mitral inflow assessment.

\section{PARTICIPANTS WITH DIABETES MELLITUS HAVE PRESERVED METABOLIC FLEXIBILITY}

Peregrine Green, William D Watson, Neil Herring, Stefan Neubauer, Oliver J Rider. Oxford Centre for Clinical Magnetic Resonance Research, Division of Cardiovascular Medicine, Radcliffe Department of Medicine, University of Oxford, UK; Department of Physiology, Anatomy and Genetics, University of Oxford, UK

\subsection{6/heartjnl-2021-BSCMR.17}

Background Measurement of the Phosphocreatine/Adenosine Triphosphate (ATP) ratio along with the Creatine Kinase (CK) rate constant $\left(\mathrm{CK}_{k f}\right)$ allows calculation of the ATP delivery rate (CK flux). Metabolic flexibility may be impaired both in heart failure with reduced ejection fraction (HFrEF) and diabetes mellitus (DM). It is unknown to what extent flexibility can be influenced by artificially altering the substrate available for metabolism.

Purpose To examine cardiac function and energetics in diabetic participants with normal cardiac function and HFrEF, clamped on either fatty acid (FA) or glucose metabolism.

Methods Participants with non-insulin dependent diabetic mellitus (NIDDM) with both normal cardiac function (NHDM) and HFrEF (HFDM) were recruited and received intravenous infusions of either Intralipid (IL) or glucose-insulin (GI) at 2 separate visits, before undergoing multi-parametric cardiac MRI at 3 Tesla. Cardiac volume and function, PCR/ATP and $\mathrm{CK}_{k f}$ were assessed. CK flux was calculated as $\mathrm{CK}_{k f} \times \mathrm{PCR} /$ ATP $\times 5.7 \quad \mu \mathrm{mol} \quad(\mathrm{g} \text { wet weight })^{-1}$ (assumed ATP concentration).

Results 15 NHDM participants (14 male, age $61.5 \pm 7.3$ years) and 9 HFDM participants (7 male, age $69.4 \pm 7.8$ years) were recruited. Left ventricular ejection fraction (LVEF) at rest was higher on IL compared to both baseline fasting and GI for NHDM (baseline 59.1 $\pm 3.8 \%$, GI 59.4 $\pm 4.3 \%$, IL $62.8 \pm 3.5 \% ; \mathrm{p}=<0.01$ ), with a non-significant trend for HFDM (baseline $37.3 \pm 7.6 \%$, GI $36.8 \pm 9.2 \%$, IL $38.8 \pm 8.0 \%$, $\mathrm{p}=0.12)$. For both NHDM and HFDM there was no difference in PCR/ATP (NHDM: GI 1.98 \pm 0.31 , IL 1.97 \pm 0.24 , $\mathrm{p}=0.99$; HFDM: GI 1.82 \pm 0.36 , IL 2.01 $\pm 0.32, \mathrm{p}=0.09$ ) or CK flux (NHDM: GI 2.6 $\pm 1.1 \mu \mathrm{mol}$ (g wet weight) ${ }^{-1} \mathrm{~s}^{-1}$, IL $1.8 \pm 1.2 \mu \mathrm{mol}$ (g wet weight) ${ }^{-1} \mathrm{~s}^{-1}, \mathrm{p}=0.08$; HFDM: GI 1.6 $\pm 1.7 \mu \mathrm{mol}(\mathrm{g} \text { wet weight) })^{-1} \mathrm{~s}^{-1}$, IL $2.3 \pm 1.1 \mu \mathrm{mol}$ (g wet weight) ${ }^{-1} \mathrm{~s}^{-1}, \mathrm{p}=0.39$ ).

Conclusion Diabetic participants with HFrEF and normal cardiac function appear to have increased resting LVEF when clamped on FA as opposed to glucose metabolism, without a significant change in energetic status. This may imply that metabolic flexibility is relatively preserved in these groups.

\section{SUBCLINICAL MYOCARDIAL INFLAMMATION IN ADULTS WITH TYPE 2 DIABETES: A CLINICAL STUDY USING MYOCARDIAL T2 MAPPING}

Pranav Ramesh, Jian L Yeo, Gaurav S Gulsin, Gerry P McCann. Department of Cardiovascular Sciences, University of Leicester and Leicester NIHR Biomedical Research Centre, Glenfield Hospital, Leicester, UK

\subsection{6/heartjnl-2021-BSCMR.18}

Background Chronic hyperglycaemia in Type 2 diabetes (T2D) results in a systemic low-grade inflammatory state. Inflammation is a key instigator in the development of heart failure in T2D. Cardiovascular magnetic resonance (CMR) T2 mapping is a technique which identifies myocardial oedema. The utility of T2 mapping to identify subclinical oedema as a marker of inflammation in T2D is unknown. We hypothesise that T2 times will be higher in subjects with T2D.

Methods CMR imaging on a 3-Tesla scanner was performed on 182 participants who were free of symptomatic cardiovascular disease. T2 images were acquired using the Siemens MyoMap sequence at the mid-ventricular short-axis slice. Twenty participants underwent a repeat CMR scan within two weeks to assess the test-retest reproducibility of T2. Intraclass correlation coefficient (ICC) and Bland-Altman plots were generated to assess reproducibility. T2 values between groups were compared using T-test or Mann-Whitney test as appropriate. Clinical determinants of $\mathrm{T} 2$ in $\mathrm{T} 2 \mathrm{D}$ were assessed using multivariable linear regression.

Results 124 T2D (mean age 64 7 , 66\% male) and 40 controls (mean age $61 \pm 8,60 \%$ male) were analysed. T2 times exhibited excellent intra-observer (ICC 0.98-0.99), moderate inter-observer (ICC 0.48-0.99), and poor test-retest variability (ICC 0.33-0.90). T2 times were significantly lower in subjects with T2D compared to controls $(39.0 \pm 2.2 \mathrm{~ms}$ versus 40.1 $\pm 2.9 \mathrm{~ms}, \mathrm{P}=0.013)$. Stratification by sex revealed significantly lower T2 in females with T2D $(39.4 \pm 2.4$ ms versus $41.7 \pm 3.1$ $\mathrm{ms}, \mathrm{P}=0.003)$, but not in males, when compared to controls. Following multivariable adjustment, T2 time was positively associated with a non-white ethnicity $(\beta=0.245, \mathrm{P}=0.007)$ and diabetic duration $(\beta=0.197, P=0.03)$ and inversely associated with systolic blood pressure $(\beta=-0.215, \mathrm{P}=0.018)$.

Conclusions T2 mapping has moderate-excellent observer variability but poor test-retest reproducibility in a cohort T2D. Lower T2 times in T2D may reflect early myocardial fibrosis but does not provide evidence of subclinical myocardial oedema and therefore is not able to detect low-grade myocardial inflammation.

\section{CARDIAC MAGNETIC RESONANCE TO IDENTIFY RAISED LEFT VENTRICULAR FILLING PRESSURE}

Rebecca Gosling, Samer Alabed, Peter Swoboda, Sherif F Nagueh, Rachel Jones, Alexander Rothman, Jim M Wild, David G Kiely, Robin Condliffe, Andrew J Swift, Pankaj Garg.

\subsection{6/heartjnl-2021-BSCMR.19}

Background Non-invasive imaging is routinely used to estimate left ventricular (LV) filling pressures (LVFP) in heart failure (HF), as an alternative to right heart catheterisation (RHC). Transthoracic echocardiography (TTE) estimates of LVFP are frequently deployed but produce largely dichotomised data limiting flexible clinical use and perform less well in patients with heart failure with preserved ejection fraction (HFpEF). 
Cardiovascular magnetic resonance (CMR) is emerging as an important imaging tool for sub-phenotyping HF. However, currently we cannot estimate LVFP from CMR. This study sought to investigate if CMR can estimate LVFP in patients with suspected HF, whether this has increased diagnostic power beyond TTE and if CMR modelled LVFP has prognostic power.

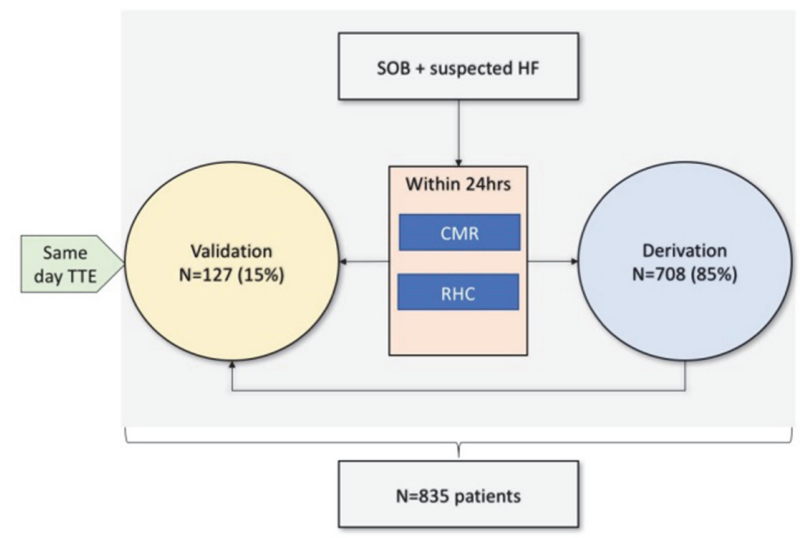

Abstract 19 Figure 1 Study flow diagram

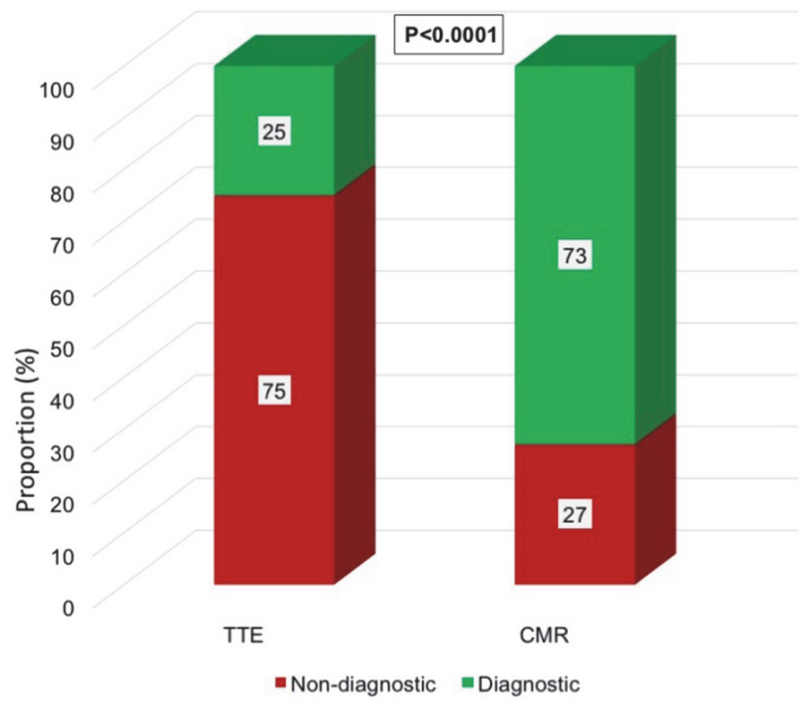

Abstract 19 Figure 2 Comparison of diagnostic accuracy of TTE and CMR derived estimates of LVFP. In the validation cohort, 75\% patients had non diagnostic TTE (incorrect classification or indeterminate) compared to $27 \%$ with CMR $(p<0.001)$

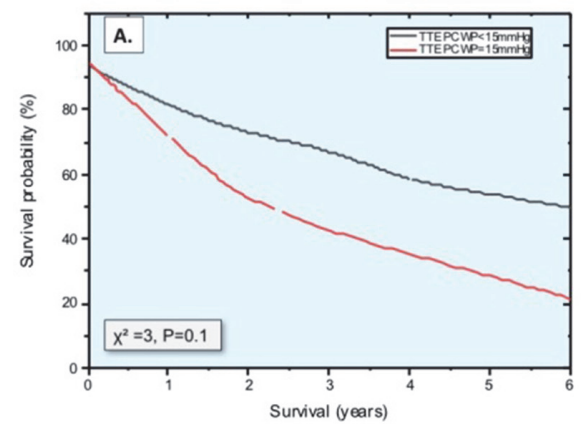

Methods Suspected HF patients underwent RHC, TTE and CMR within 24 hours of each other. RHC measured pulmonary capillary wedge pressure (PCWP) was used as a reference for LVFP. CMR included left/right heart volumetric assessment and left atrial area. Patients were split into derivation (85\%) and validation (15\%) cohorts (figure 1). In the derivation cohort, multivariate regression was used to determine predictors of LVFP. The CMR-derived model was then applied to the validation cohort and diagnostic accuracy was compared with TTE. Association of CMR modelled LVFP with mortality was determined using Kaplan-Meier (KM) survival analysis.

Results We enrolled 835 patients (mean age 66 13 years, $38 \%$ male). Two CMR metrics were incorporated in the final model; LV mass and left atrial area. When applied to the validation cohort, CMR modelled PCWP had good correlation with RHC PCWP $(\mathrm{R}=0.6)$. The diagnostic accuracy of CMR modelled PCWP to predict elevated filling pressures (RHC PCWP > 14 mmhg) was 73\%. TTE was non-diagnostic in $75 \%$ of cases (incorrect classification or indeterminate result). Of these, $71 \%$ were reclassified to a correct diagnosis by CMR (figure 2). CMR modelled PCWP was identified as an independent predictor of death on $\mathrm{KM}$ analysis (HR 2.18 (95\% CI 1.1 to 4.3 ), $\mathrm{P}=0.02$ ) (figure 3).

Conclusion A physiological CMR model can estimate LVFP in patients with suspected HF. Our model demonstrated good diagnostic accuracy providing additive value to TTE assessment. In addition, CMR modelled LVFP has a prognostic role.

\section{APICAL ISCHAEMIA IS UBIQUITOUS IN APICAL HYPERTROPHIC CARDIOMYOPATHY AND OCCURS BEFORE OVERT HYPERTROPHY}

1,2 Rebecca K Hughes, 1,2 João B Augusto, ${ }^{1,2}$ Kristopher Knott, ${ }^{1,2}$ Andreas Seraphim,
${ }^{1,2}$ George Joy, ${ }^{2,3}$ Saidi Mohiddin, $1,4,5$ Gabriella Captur, 1,2 Luis R Lopes, ${ }^{6}$ Peter Kellman,
1,2 James C Moon. 'Institute of Cardiovascular Science, University College London, Gower
Street, London, UK; ${ }^{2}$ Barts Heart Centre, The Cardiovascular Magnetic Resonance Imaging
Unit and The Inherited Cardiovascular Diseases Unit, St Bartholomew's Hospital, West
Smithfield, London, UK; ${ }^{3}$ William Harvey institute, Queen Mary University of London, UK;
${ }^{4}$ Inherited Heart Muscle Conditions Clinic, Gower Street, Department of Cardiology; ${ }^{5}$ Royal
Free Hospital, NHS Trust, UK; ${ }^{6}$ University College London MRC Unit of Lifelong Health and
Ageing, 1-19 Torrington Place, Fitzrovia, London, UK; ${ }^{7}$ National Heart, Lung, and Blood
Institute, National Institutes of Health, DHHS, Bethesda, Maryland, USA

10.1136/heartjnl-2021-BSCMR.20

Background Small vessel disease and associated microvascular ischaemia is a key feature of hypertrophic cardiomyopathy (HCM). Perfusion defects are described in $41-79 \%$ of cases ${ }^{1,2}$. Apical HCM (ApHCM) has features suggesting distinction

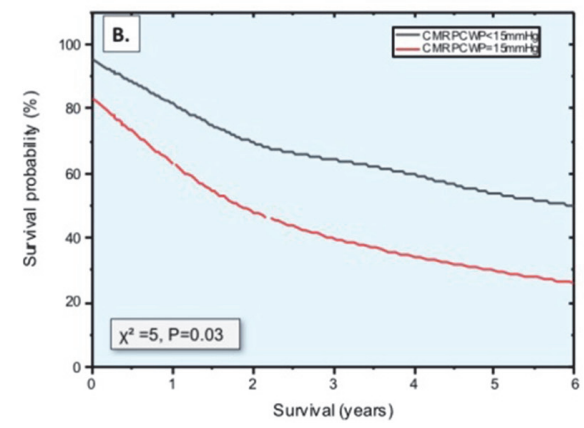

Abstract 19 Figure 3 Kaplan-Meier survival curves of patients with normal versus elevated LVFP as determined by (a) TTE and (b) CMR 